\title{
The wider costs of large roads on health and wellbeing
}

Paulo Rui Anciaes, Peter Jones, Jemima Stockton, Jennifer S. Mindell

University College London

\section{COMMUNITY SEVERANCE (BARRIER EFFECT)}

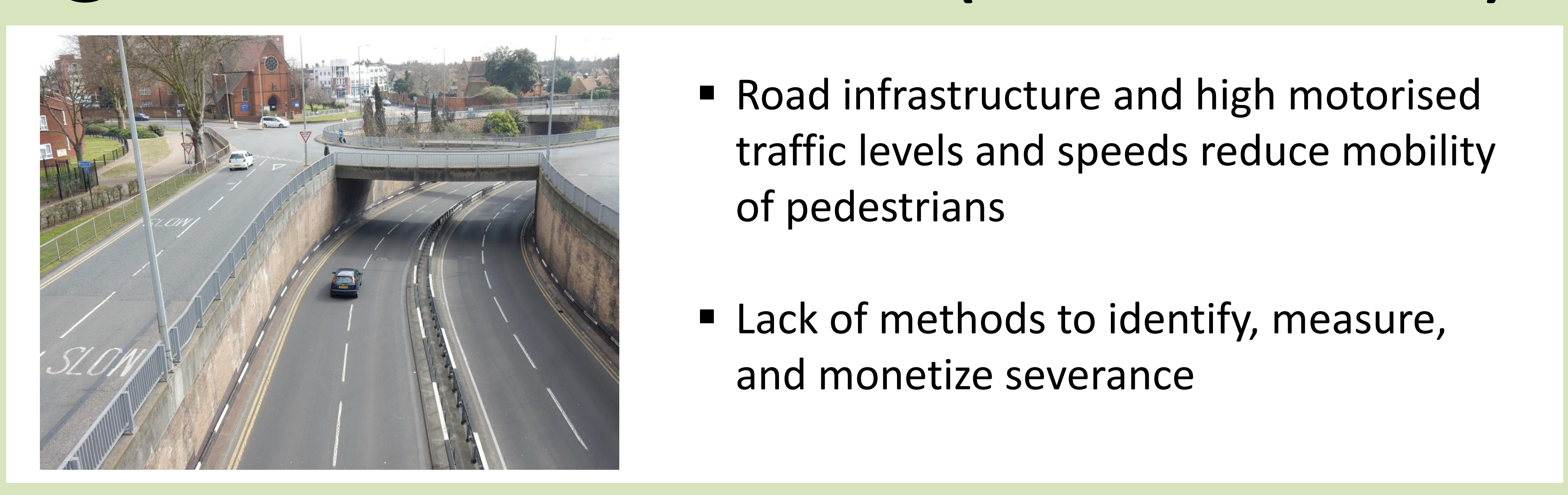

\section{DIRECT EFFECTS}

A. Stated preference survey

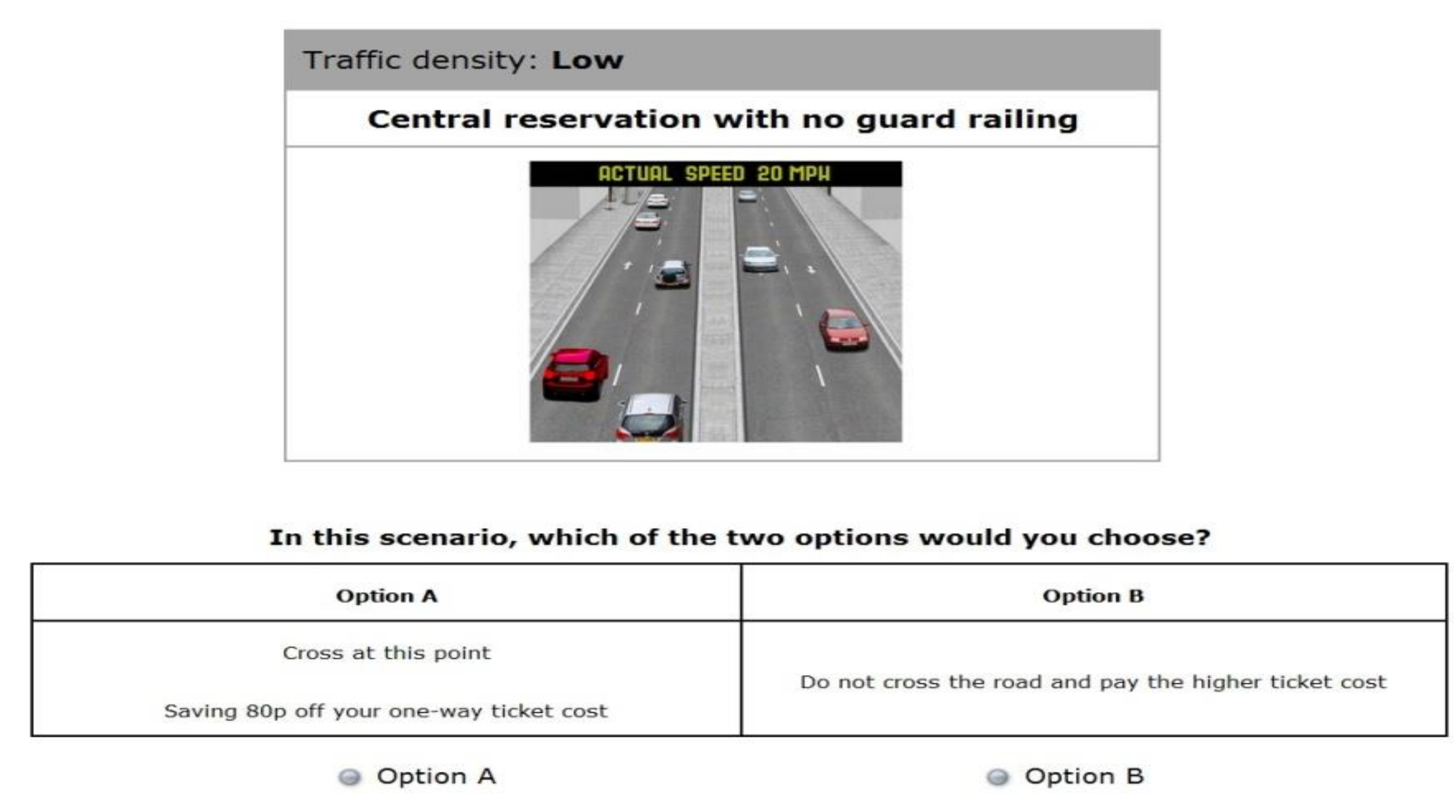

Each participant answers 8 questions, with different number of road lanes, median strip $(\mathrm{Y} / \mathrm{N})$, traffic levels, traffic speeds, and value of cost saving

B. Random-effects logit model - Positive

Dep. var: probability of crossing the road vs. not crossing 1 - Negative

\begin{tabular}{lcc} 
& coeff. $\rightarrow$ Trade-off value with saving (f) \\
\hline Constant & $\bullet$ & \\
Saving & $\bullet$ & \\
Lanes=3 & $\bullet$ & 1.63 \\
No medium strip & $\bullet$ & 1.47 \\
Density=medium & $\bullet$ & 1.10 \\
Density=high & $\bullet$ & 2.45 \\
Speed=30mph & $\bullet$ & 0.50 \\
\hline
\end{tabular}

Ex: On average, people are willing to forego a saving of $f 2.45$ in order to avoid crossing a road with high traffic density (one time)

\section{A NEW METHOD TO MONETIZE SEVERANCE}

A. Direct effects (on pedestrians)

delay, collision risk, inconvenience of crossing the road

$\rightarrow 3$

B. Wider effects (on travel behaviour)

- Do not travel $\rightarrow$ increased risk of social exclusion

- Do not walk $\rightarrow$ less physical activity

- Travel by car $\rightarrow$ external effects of motorised traffic

$\rightarrow 4$

\section{WIDER EFFECTS}

A. Household survey

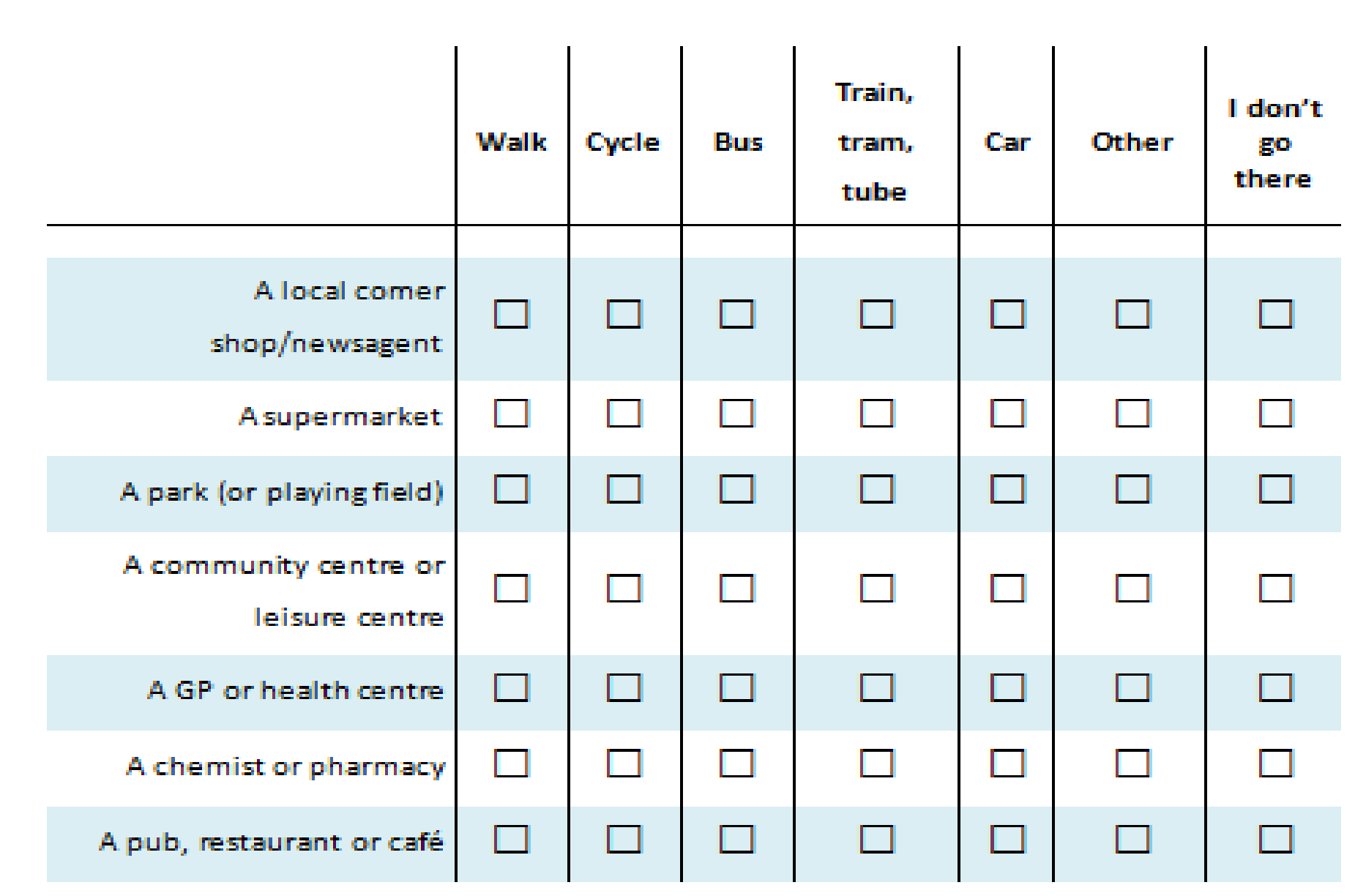

AND rate the amount of traffic on the busiest road near you

\section{B. Multinominal} logit model

Dep. vars: probability of travelling
by a given mode vs. not travelling

Omitted categories: shop, low traftic
volume, not walking distance, etce

Coefficients:

- Negative

Insignificant not shown

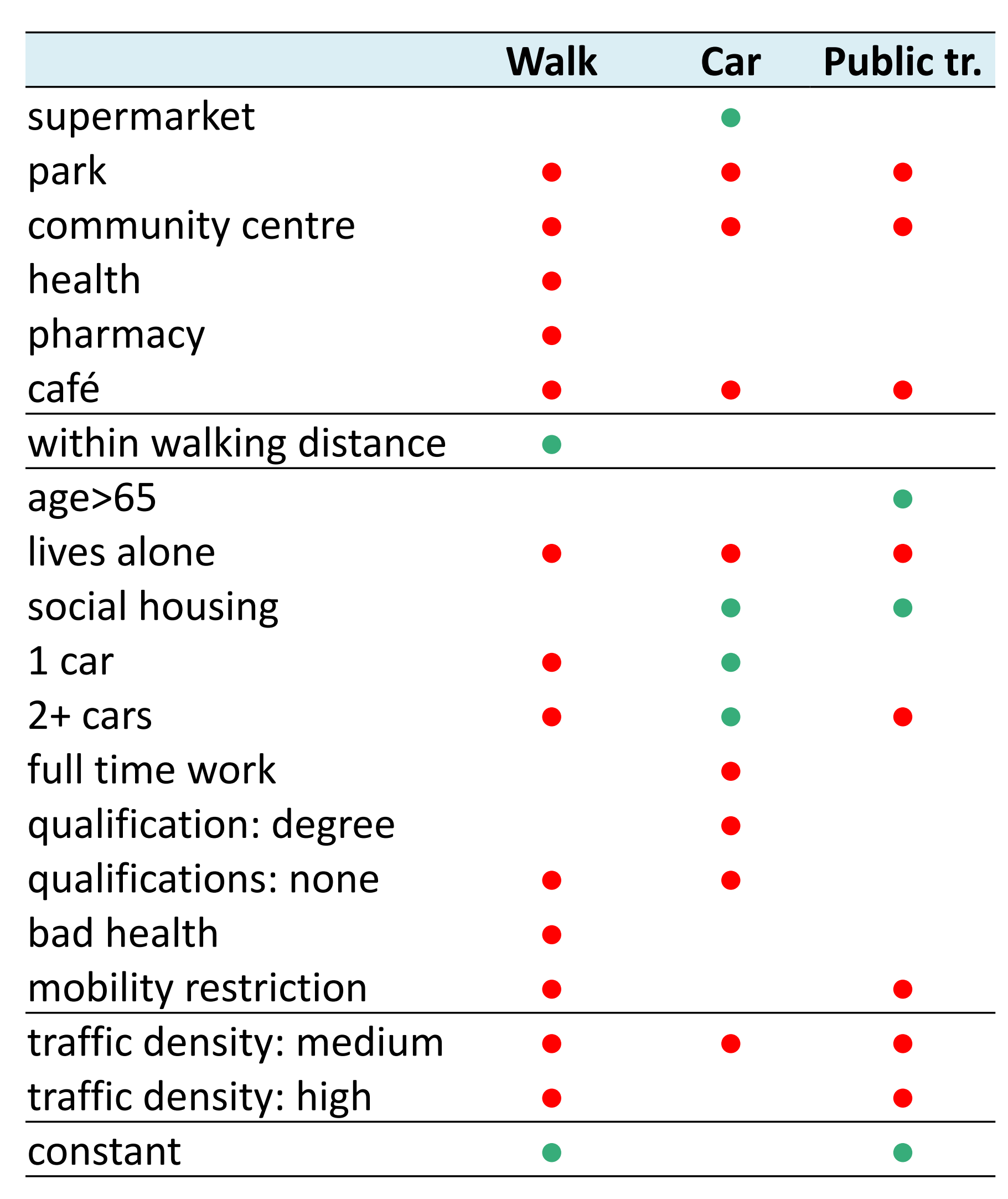

C. Effect of high traffic levels on probability of each choice (vs. low traffic levels)

\begin{tabular}{lcccc}
\multicolumn{1}{c}{ Place } & Walk & Car & Other & Don't Travel \\
\hline shop & $-2.2 \%$ & $1.4 \%$ & $0.1 \%$ & $0.6 \%$ \\
supermarket & $-5.2 \%$ & $4.8 \%$ & $-0.2 \%$ & $0.7 \%$ \\
park & $-8.7 \%$ & $1.5 \%$ & $-1.1 \%$ & $8.2 \%$ \\
community centre & $-10.8 \%$ & $0.6 \%$ & $-1.7 \%$ & $11.9 \%$ \\
health & $-5.5 \%$ & $3.5 \%$ & $-0.2 \%$ & $2.2 \%$ \\
pharmacy & $-4.6 \%$ & $2.8 \%$ & $0.0 \%$ & $1.8 \%$ \\
café & $-7.0 \%$ & $8.4 \%$ & $-2.3 \%$ & $0.8 \%$ \\
\hline
\end{tabular}

\section{Effect on number of trips}

(per year per person)

National travel survey (England)

(avg. number of trips/year/person )

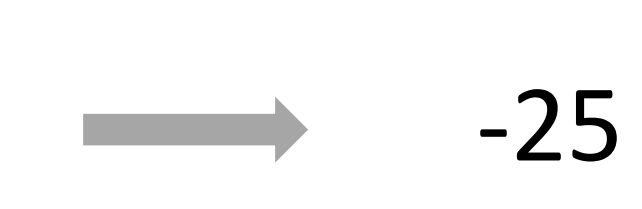

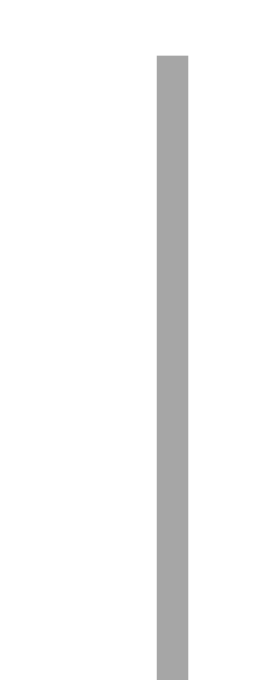

E. Costs

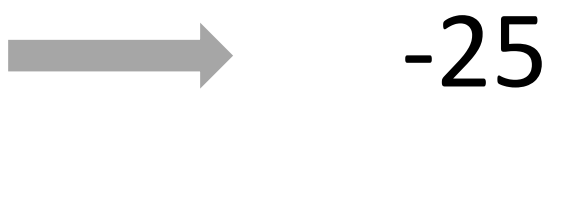

$+15$

$+12$

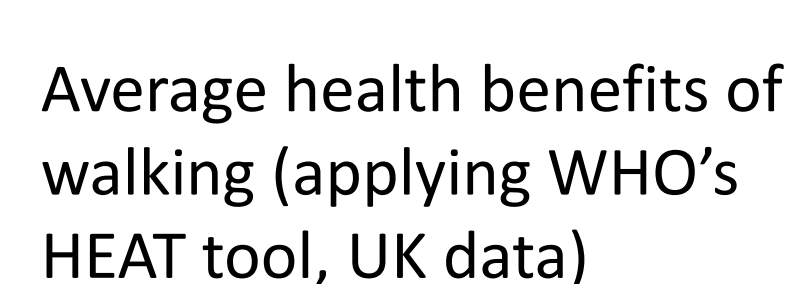

$*$ walking (applying W
HEAT tool, UK data)

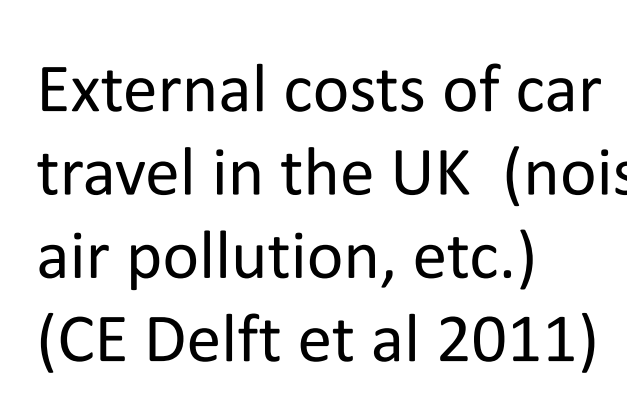
Trade-off between trips and income
for someone at risk of social
exclusion (Stanliey et al 2012) $=$ $=$

$£ 38.00$

f1.98

f11.59

Ex: The health costs of the reduction of walking trips cause by high traffic densities (comparing with low densities) are f38/year/person
5 CONCLUSIONS :

People attach a monetary value to avoid crossing a busy road. That value is an indicator of the disutility caused by motorised traffic on pedestrians High traffic densities decrease the probability of walking and increase the probability of using car or not travelling. The monetary values of the
Street Mobility and Network Accessibility

Developing tools to identify and overcome barriers to walking y @s $\overline{\text { EPSRC }}$ 